\title{
New approach for estimate parameters of machine asynchronous
}

\author{
Abdelmalik BOUHENTALA ${ }^{1}$, Mohamed Salah BENBOUZA ${ }^{2}$ \\ 1,2 (Department of Electrical Engineering Faculty of Science, University of Batna Algeria.)
}

\begin{abstract}
Rotating electrical machines play an important role in the industrial world. Among them, the threephase squirrel cage asynchronous machines are frequently used because of their robustness, their relatively simple construction and their low cost. Nevertheless, during their lifespan, these machines are submitted to external and internal stresses which can lead to several failures. In this paper, we present the estimate of the parameters sensitive to the defects, namely the rotor currents and resistances of the rotor bars, by using the Extended Kalman filter.
\end{abstract}

Keywords: Asynchronous machine, multi-winding model, extended Kalman filter, break bar.

\section{Introduction}

The fault diagnosis of electrical machinery gained special importance due to loss of downtime and revenue machine industry. Often, these machines run critical loads and their sudden failure can be catastrophic. Thus, the drive system of the engine should also have the diagnostic features to predict defects machine their very beginning. Consequently, it becomes very important to have models of machine and control techniques that can distinguish the healthy and faulty machines [1]. [3]. [6]. Among the stochastic methods, we have the Kalman filter. A special case of this method is the Extended Kalman Filter. It is suitable for the treatment of discrete and noisy measurements to obtain accurate estimates of state variables and model parameters. The general way in which it does is to use the following factors: -Knowledge of system dynamics and measurements.-The statistical description of noise system, disturbances, measurement errors and uncertainties of system model. To evaluate these methods on a single representative example, we present in this paper, the estimation algorithm based online the extended Kalman filter for parameter estimation of induction machine in view of detection rotor faults.For the detection of rotor faults in the approach based on parametric models, it really does not generate a vector of residuals, but estimates a vector of parameters whose variation outside a reference range represents the occurrence of a fault in the monitored system (or more accurately represents a change in the characteristics of the process). This variation can then be detected using a test of decision in the parameter space [2] [4].The algorithm is verified by simulation. Consider an asynchronous machine. The measurable variables are the phase currents, phase voltages and rotor speed.

\section{Discrete models}

The estimated state variable for the detection of defects requires a dynamic model of multi-windings adequacy of induction machine. The model is obtained using the transformation PARK. This model raises the usual assumptions [6]. The Kalman filter requires a discrete model of the machine that is deducted from the continuous model. The representation of the dynamics of the machine with a marker binds to the rotor is given by the following equations:

$$
\begin{array}{ll}
\mathrm{Vds}=\mathrm{Rs} . \mathrm{Ids}+\frac{d \Phi d s}{d t}-\mathrm{Ws} \Phi q s \quad \text { (1); } & \mathrm{Vqs}=\mathrm{Rs} \mathrm{Iqs}+\frac{d \Phi q s}{d t}+\mathrm{Ws} \Phi d s \\
0=\mathrm{RrIdr}+\frac{d \Phi d r}{d t} & (3) ; \quad 0=\mathrm{RrIqr}+\frac{d \Phi q r}{d t}
\end{array}
$$

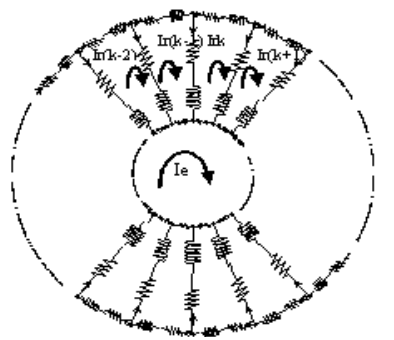

Fig.1. Electrical equivalent circuit of a cage rotor. 
By choosing isd, isq, ird, irq,Ie as state variables of the model schema multi-machine winding asynchronous representation in state space, the complete system $[L] \frac{d[I]}{d t}=[V]-[R][I]$, after processing Park method, we have led model:

$$
\begin{aligned}
& \dot{x}=A \cdot x(t)+B \cdot u(t) \\
& x(t)=\left[\begin{array}{lllll}
i s d(t) & i s q(t) & i r d(t) & i s q(t) & I e(t)
\end{array}\right]^{T} \\
& u(t)=\left[\begin{array}{llll}
V s d & V s q & 0 & 0
\end{array}\right]^{T}
\end{aligned}
$$

The out equation is:

$$
z(t)=h[x(t), t]
$$

Where: $h=[$ isd isq $]$

The model is deterministic:

$$
\frac{d}{d t}\left[\begin{array}{c}
I d s \\
I q s \\
I d r \\
I q r \\
I e
\end{array}\right]=\left[\begin{array}{ccccc}
-a 1 & a 2 . w & -a 3 . S 1 & a 4 & 0 \\
-a 6 . w & -a 7 & a 8 & a 9 . S 1 & 0 \\
-a 11 & a 12 . w & -a 13 . S 1 & -a 14 & 0 \\
a 16 . w & a 17 & -a 18 & -a 19 . S 1 & 0 \\
0 & 0 & 0 & 0 & L e
\end{array}\right]\left[\begin{array}{c}
I d s \\
I q s \\
I d r \\
I q r \\
I e
\end{array}\right]+\left[\begin{array}{cc}
a 5 & 0 \\
0 & a 10 \\
a 15 & 0 \\
0 & -a 20 \\
0 & 0
\end{array}\right]\left[\begin{array}{c}
V d s \\
V q s \\
0 \\
0 \\
0
\end{array}\right]
$$

$a 1=a 7=4 . m 1$. Lrc.$R s ; a 2=a 6=4 . m 1 . L r c . L r c ; a 3=a 9=2 . m 1 . M s r . N r ; a 4=($ Lrc $. \omega-S 2) \cdot 2 . m 1 . M s r . N r$ $a 5=a 10=4 . m 1 . L r c ; a 8=(\operatorname{Lrc} . \omega+S 3) \cdot 2 . m 1 . M s r . N r ; a 11=a 17=6 . m 1 . M s r . R s$

$a 12=a 16=6 . m 1 . M s r . L s c ;{ }_{a 13}=a 19=\left(3 . M s r^{2} . N r+1\right) \cdot\left(\frac{1}{L r c}\right) ; a 15=a 20=6 . m 1 . M s r$

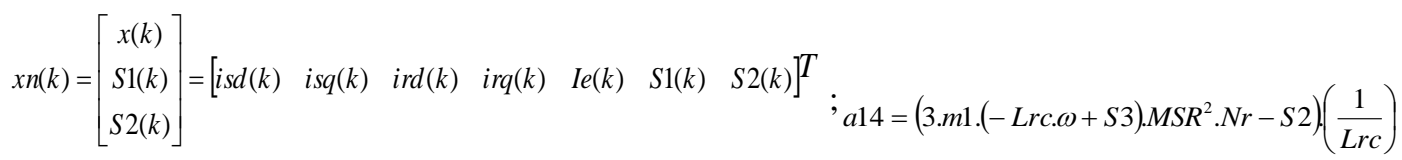

$a 18=\left(3 \cdot m 1 \cdot(L r c \cdot \omega+S 2) \cdot M S R^{2} \cdot N r-S 2\right) \cdot\left(\frac{1}{L r c}\right) ; m 1=\frac{1}{\left(-3 \cdot M s r^{2} \cdot N r+4 \cdot L r c \cdot L s c\right)}$

$S 1=\frac{2}{16}\left[\left(2 \frac{R e}{N r}+R b 0+R b 15\right) \cos ^{2} 0 \alpha+\left(2 \frac{R e}{N r}+R b 1+R b 0\right) \cos ^{2} 1 \alpha+\cdots+\left(2 \frac{R e}{N r}+R b 14+R b 15\right) \cos ^{2} 15 \alpha\right]-\frac{4}{16}[(R b 0 \cos 0 \alpha \cos 1 \alpha)+(R b \cos 1 \alpha \cos 2 \alpha)+\cdots+(R b 15 \cos 15 \alpha \cos 0 \alpha)]$

$S 2=-\frac{2}{16}\left[\left(2 \frac{R e}{N r}+R b 0+R b 15\right) \cos 0 \alpha \sin 0 \alpha-\left(2 \frac{R e}{N r}+R b 1+R b 0\right) \cos 1 \alpha \sin 1 \alpha-\cdots-\left(2 \frac{R e}{N r}+R b 14+R b 15\right) \cos 15 \alpha \sin 15 \alpha\right]+$

$\frac{2}{16}[(R b 0 \sin 0 \alpha \cos 1 \alpha)+(R b 1 \sin 1 \alpha \cos 2 \alpha)+\cdots+(R b 15 \sin 15 \alpha \cos 0 \alpha)]$

$+\frac{2}{16}[(R b 0 \cos 0 \alpha \sin 1 \alpha)+(R b 1 \cos 1 \alpha \sin 2 \alpha)+\cdots+(R b 15 \cos 15 \alpha \sin 0 \alpha)]$

(12)

Where: S1, S2, represents the equivalent resistance of rotor bars.

We have in these equations, $\mathrm{A}$ and $\mathrm{B}$ are the matrices of the continuous model and $\mathrm{AD}, \mathrm{BD}$ are the matrices corresponding discrete model. $\mathrm{k}$ represents the discretized time instants and $\mathrm{Te}$ is the sampling period.

\subsection{Enhanced Discrete model}

The matrix A varies over time to elements that depend on the rotor speed. When parameters, in this case the equivalent resistance S1, S2, are unknown, another equation must be added to the model of the machine, the equation of state is no longer linear. To estimate the equivalent resistance, we introduce the equations:

$\mathrm{S} 1(\mathrm{k}+1)=\mathrm{S} 1(\mathrm{k})$

$\mathrm{S} 2(\mathrm{k}+1)=\mathrm{S} 2(\mathrm{k})$; This equation is based on assumption that the equivalent resistances are not varied during the

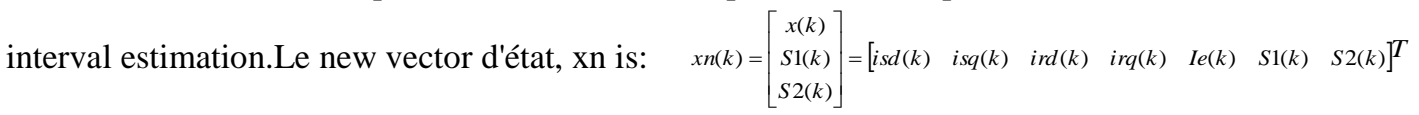

The out equation is : $z(k)=\left[\begin{array}{c}i s d(k) \\ i s q(k)\end{array}\right]$ 


\subsection{Stochastic Model}

In practice, the asynchronous machine cannot be modeled so perfect. This is due to assumptions in the model dynamics and errors that occur in measurements, filters, sensors, etc. The use of approximations increases errors compared to the values of stator currents. We consider all these errors and uncertainties such as noise in the measurements and the model structure. We assume in our study that the noise can be approximated by the Gaussian distribution law.

\subsection{Extended Kalman filter.}

The Kalman filter $(\mathrm{KF})$ is a linear estimation technique. It cannot be used for estimating state variables and parameters of a nonlinear system unless the system model is linearized around an operating point. This linearization procedure is known as the extended Kalman filter [7] [8] [9]. Then, the algorithm of Kalman filter is conventional as used to estimate simultaneously the system states and parameters of the machine simultaneously. The filter has a structure preacher correction, The state time $(\mathrm{k}+1)$ depends not only on the state at time (k), but also the model error $\mathrm{w}(\mathrm{k})$. Since these errors are unknown, knowledge of the mathematical model can give us only the prediction of the state at the moment $(\mathrm{k}+1)$. The estimates xne(k) can be obtained recursively using the measures to improve the predictive variables. The correction is proportional to predictions residue steps:

\subsection{Application of Extended Kalman filter has the asynchronous}

The algorithm described above is applied to the simulation study for estimating parameters of a machine

$$
\begin{aligned}
& F(k)=\left[\begin{array}{ccc}
A D(k) & F 1(k) & F 5(k) \\
& F 2(k) & F 6(k) \\
F 3(k) & F 7(k) \\
F 4(k) & F 8(k) \\
1 & 1
\end{array}\right] \\
& F 1(k)=\frac{\partial d i d s}{\partial S 1}, F 2(k)=\frac{\partial d i q s}{\partial S 1}, F 3(k)=\frac{\partial d i d r}{\partial S 1}, F 4(k)=\frac{\partial d i q r}{\partial S 1} \\
& F 5(k)=\frac{\partial d i d s}{\partial S 2}, F 6(k)=\frac{\partial d i q s}{\partial S 2}, F 7(k)=\frac{\partial d i d r}{\partial S 2}, F 8(k)=\frac{\partial d i q r}{\partial S 2}
\end{aligned}
$$

\section{Simulation results.}

Considering the difficulty of seeing the empty defects, we will simulate the machine supported with break bar. The figure (2) illustrates the temporal evolution of transient and steady speed of rotation, it stabilizes at a value of $2219 \mathrm{tr} / \mathrm{mn}$. This figure also illustrates the electromagnetic torque Cem presence of rupture bars rb0 at $\mathrm{t}=1 \mathrm{~s}$. After a transitional phase, it stabilizes at a value close to $3.5 \mathrm{~N}$ due to the presence of load torque.The figure (3) illustrates the temporal evolution of transient and steady flow of bars $r b 0$ à $t=1 s$, with their current estimates in the presence of a break bar rb1 à $\mathrm{t}=1 \mathrm{~s}$.. The currents flowing in the bars adjacent to broken bars are well above their face value. The comparison of estimated and measured current shows good convergence of the filter.

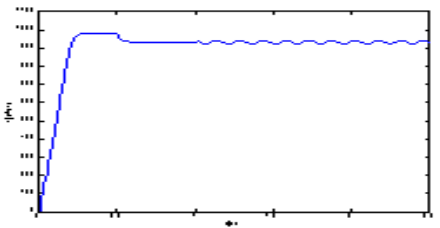

Fig.2(a)

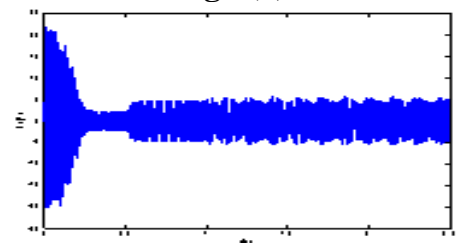

Fig.3.

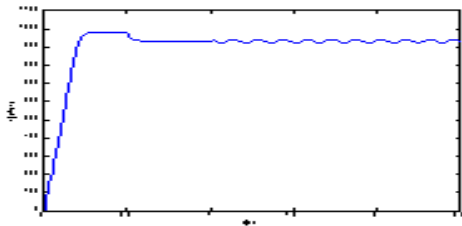

. Fig.2(b)

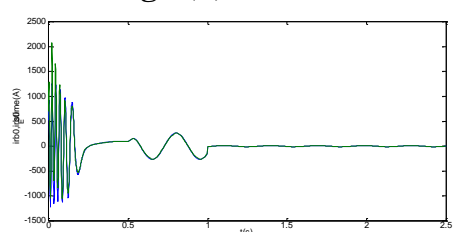

Fig.4(a)

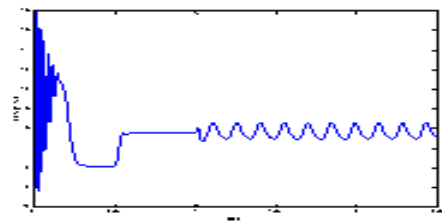

Fig.2(c)

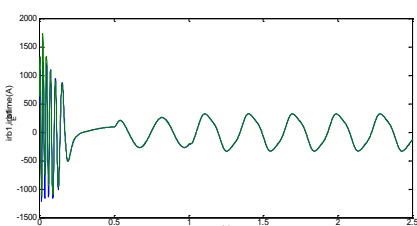

Fig.4(b).

It now imposes a torque load of $3.5 \mathrm{Nm}$ at $\mathrm{t}=0.5 \mathrm{~s}$ and the machine then operates with fracture load of two bars.The figure (5) illustrates the temporal evolution of transient and steady electromagnetic torque Cem presence of a break bar $r b 0 \mathrm{at}=1 \mathrm{~s}$ et $\mathrm{rb} 1 \mathrm{at}=2 \mathrm{~s}$. After a transitional phase, it stabilizes at a value close to 3.5 
N.m ,because of the presence of load torque, we can see in this figure ripples in gait speed and torque, is due to breakage of the bars. The figure (6) illustrates the temporal evolution of transient and steady stator current in the presence of a break bar rb0 à $\mathrm{t}=1 \mathrm{~s}$. The figure (7) illustrates the temporal evolution of transient and steady flow of bars irb1, irb2 and their current estimated in the presence of a bar break rb0 à $t=1 \mathrm{~s}$ et à $\mathrm{t}=2 \mathrm{~s}$, or we see the convergence of the filter. The currents flowing in the bars adjacent to broken bars are well above their face value. We therefore conclude that there is a risk of failure in each since the thermal and electrical stresses are redistributed to the adjacent conductors. The preceding figures are the equivalent resistance of rotor bars observed. After a period of convergence, the observed resistance converges to the same resistance. We notice a slight oscillation around this value.

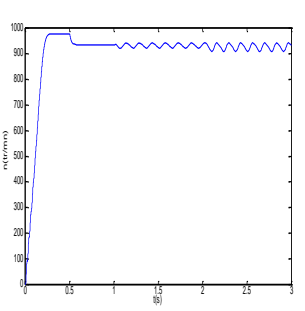

Fig.5

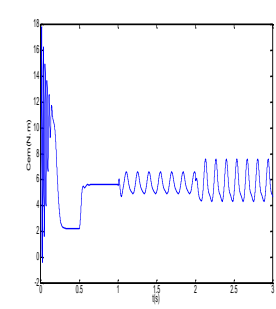

Fig.6

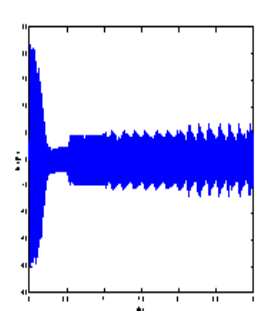

Fig.7(a)

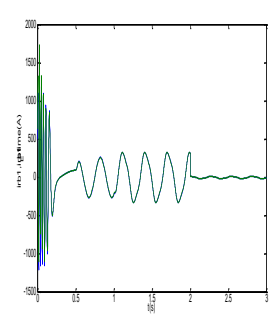

Fig.7(b)

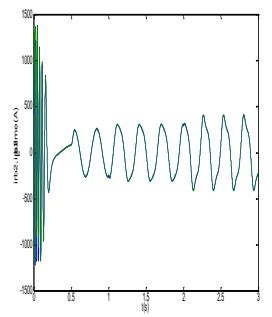

Fig.7(c
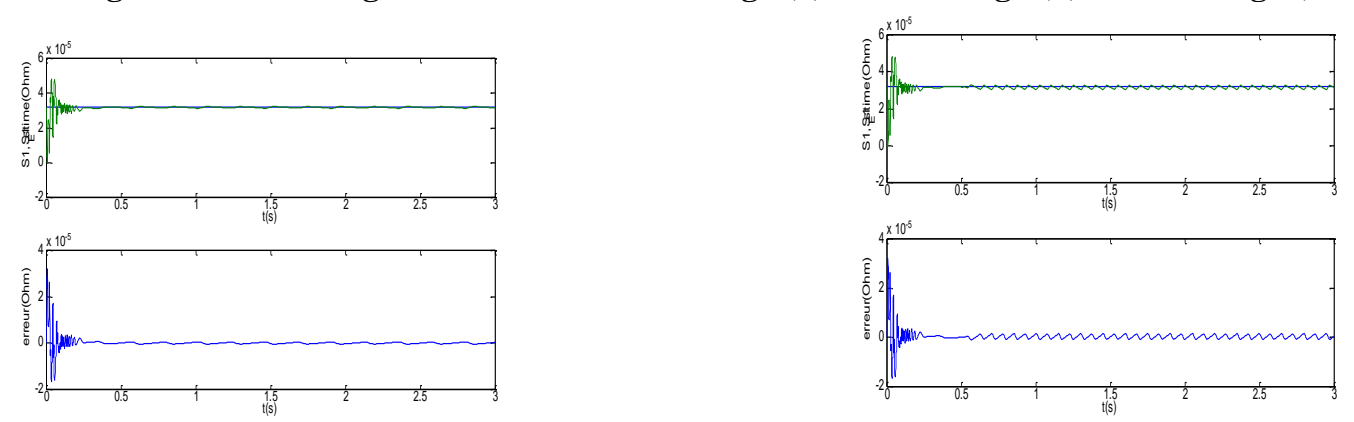

Fig.8. Estimated rotor resistance equivalent S1,
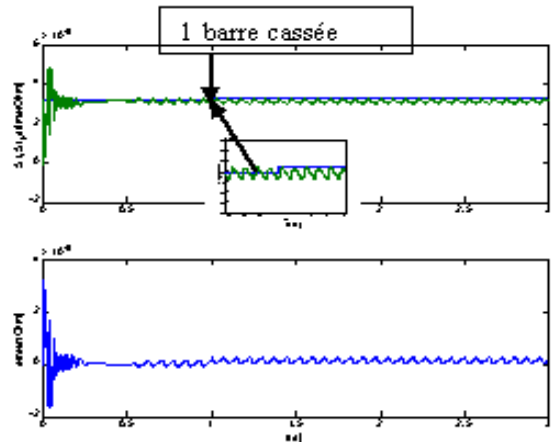

Fig.(10)

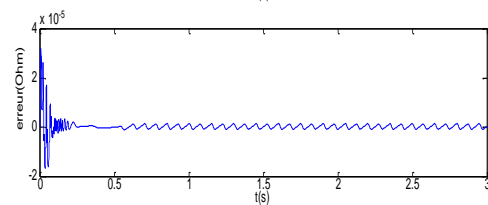

Fig.9 Estimated rotor S1, Cr=3.5N.m.
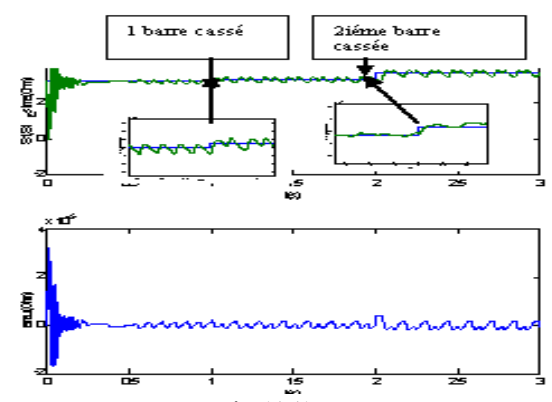

$\operatorname{Fig}(11)$

Fig.10. Estimated rotor resistance equivalent S1, machine default (break one bar) and charge Cr=3.5N.m.

Fig.11. Estimated rotor resistance equivalent $\mathrm{S} 1$, machine default (break two bars) and charge $\mathrm{Cr}=3.5 \mathrm{~N} . \mathrm{m}$.

The previous figures show the equivalent resistance of rotor bars observed. After a period of convergence, the observed resistance converges to the same resistance. We notice a slight oscillation around this value.

\section{Change settings.}

In practice, the parameters of the asynchronous machine, other than S1, are not known exactly and estimate the result of an identification system made, for example, methods for estimating off-line. These parameters are necessary for calculating the discrete model of the machine used by the Kalman filter. In addition, the parameters of the induction machine may change due to natural phenomena heating, magnetic saturation...Two problems then arise in the calculation of the Kalman filter:

The variation of physical parameters of the synchronous machine (non-stationary behavior) and The parametric uncertainty in the discrete model due to error in identifying prior 


\section{Conclusions}

We have described a technique to estimate and follow the currents and the equivalent rotor resistance rotor bars of model schema multi-windings of the induction machine. This technique has been verified by simulation. The results obtained show that the extended Kalman filter can accurately estimate in normal operation of the machine, and in the presence of rotor faults (broken bar).The advantage of using the stator current as state variables is that they are directly measurable. The simulation with MATLAB to provide results that reflect the state of the machine. Our filter allows us to identify the presence of a defect in the rotor. We can distinguish the physical defect that alters engine operation.

\section{References}

[1]. Bachir.S, Contribution au diagnostic de la machine asynchrone par estimation paramétrique, thèse de doctorat, Ecole supérieure d'Ingénieurs de Poitiers, décembre 2002.

[2]. Boumegoura. T, Recherche de signature électromagnétique des défauts dans une machine asynchrone, et synthèse d'observateurs en vue du diagnostic, thèse de doctorat, l'école doctorale électronique, électrotechnique, automatique de Lyon, Mars 2001.

[3]. HENRY. D, Diagnostic et contrôle de cohérence des systèmes multivariables incertains, Novembre 1999, l'Université Bordeaux I.

[4]. Devanneaux.V , Modélisation des machines asynchrones triphasées à cage d'écureuil en vue de la surveillance et du diagnostic, Thèse de doctorat en génie électrique, Institut National Polytechnique de Toulouse, décembre 2002.

[5]. Bulent ,Ayhan. Mo-Yuen Chow, and Myung-Hyun Song, ,Multiple Signature Processing-Based Fault Detection Scheme for Broken Rotor Bar in Induction Motors, IEEE Transactions on energy conversion vol. 20, NO.2, june 2005

[6]. Baghli, L, Contribution à la commande de la machine asynchrone, utilisation de la logique floue, des réseaux de neurones et des algorithmes génétiques, thèse de doctorat, université Henri Poincaré, Nancy, janvier 1999.

[7]. Bandyopadhyay, M.N Sharma, N., Prakash, R, Extended Kalman Filter Approach to Joint State and Parameter Estimation, IE(I) Journal-ID (India),pp 19-23,Vol 84, November 2003

[8]. Durieu,C. Loron,L. Sedda,E. Zein, I, Fault Detection of an Induction Motor by Setmembership Filtering and Kalman Filtering ,ECC'99, September 1999, Karlsruhe.

[9]. Zein, I, Application of the Kalman Filter andLuenberger observer to the control of the inductionmotor, Thesis in Systems Control, Université de Technologie de Compiègne, Sep. 